\title{
Emergency centres lack defibrillator knowledge
}

\author{
Pauline Louw, David Maritz, Lee Wallis
}

To the Editor: In the emergency centre (EC), a number of essential items of equipment are needed to manage a wide variety of acute life-threatening emergencies. Their correct use depends heavily on the training and experience of personnel.

The defibrillator is part of this essential equipment and should be available in all ECs. Although defibrillators are widely available in Western Cape ECs, it is not known whether public sector EC personnel have the knowledge and skills necessary to use them safely and effectively.

Most cardiac arrests in adult patients are due to ventricular fibrillation (VF) or cardiac-related causes, and early defibrillation improves survival of such patients ${ }^{1,2}$ and is therefore an important part of their immediate treatment (combined with the other links of the chain of survival for both in- and out-of-hospital cardiac arrest). ${ }^{1}$

\section{Aims}

We aimed to assess the availability of defibrillators, and the current level of the correct and effective use of defibrillators among health care personnel in Western Cape public sector ECs, by means of a questionnaire (reproduced in an appendix to the web version of this article).

\section{Methods}

This was a prospective, cross-sectional study including all public hospitals with an EC in the Western Cape. Private hospitals and primary health care facilities (including community day centres and community health centres) and hospitals that refused to participate or failed to provide fully completed questionnaires were excluded.

Ethical approval was obtained from the University of Cape Town Research Ethics Committee.

Following agreement to participate, all full-time personnel in the hospital's EC were approached with a questionnaire comprising various questions including job description of personnel member completing the questionnaire, questions specific to defibrillators and pacing in the EC, and questions on defibrillators and pacing in general.

Data were collected on a Microsoft Excel database (Redmond, Va, 2007), on a password-protected work computer. Data analysis was performed using SAS Version 9.1.3.

Division of Emergency Medicine, University of Cape Town and Stellenbosch University, Tygerberg, W Cape

Pauline Louw, MB ChB, MMed (EM)

David Maritz, MB ChB

Lee Wallis, MB ChB, MD, Dip Sport Med, FRCS Edin (A\&E), FCEM, FCEM (SA)

\section{Results}

In the public health sector of the Western Cape, 3 central, 4 regional and 33 district hospitals (7 in the city, 26 rural) provide ECs. All 40 public sector hospitals with an EC in the Western Cape were approached; 2 refused to participate and 11 did not return the questionnaire forms in time, so 27 hospitals (67.5\%) were analysed.

Of the 378 forms retrieved, 12 (3.2\%) were excluded owing to lack of signed consent and 366 were analysed. The response rate varied by institution from $11.5 \%$ to $100 \% ; 68 \%$ of forms were completed by nursing personnel.

Of the hospitals that participated in the study, 22 (81.5\%) were district level, $3(11.1 \%)$ regional and 2 (7.4\%) central hospitals. All 27 hospitals had a defibrillator present in the EC, the number varying from 1 (16 hospitals, 59.3\%) to more than 3 (1 hospital, 3.7\%). Defibrillator specification varied between facilities: $67 \%$ had biphasic, $15 \%$ monophasic and $18 \%$ both mono- and biphasic defibrillators in the EC. Thirty-seven per cent of hospitals had pacing capabilities in the EC - all the hospitals without pacing were district level.

Defibrillator tests occur in $67 \%$ of ECs, but frequency of testing varied widely. Although $52 \%$ of defibrillators were tested daily, only $26 \%$ of these tests were documented in a dedicated $\log$.

Of the participants, $58 \%$ had no previous training in using a defibrillator. Only $13 \%$ of nurses and $30 \%$ of sisters had received training, compared with $88 \%$ of doctors. Of the participants $96 \%$ knew that a defibrillator was present in their EC; however, only $42 \%$ knew which type of defibrillator it was, and only $58 \%$ were correctly aware that their EC had pacing capabilities. An alarming 58\% of personnel had not used a defibrillator before, although $88 \%$ had witnessed its use. Only $17 \%$ of personnel had paced a patient before.

Areas of knowledge tested were correct use of Joule settings, indications for defibrillation, and indications for synchronised cardioversion. Only $14 \%$ of EC personnel were aware of the correct Joule setting for their defibrillator, only $18 \%$ knew all the indications for defibrillation, and only $6 \%$ were aware of the correct indications for synchronised cardioversion. Fig. 1 shows the differences in defibrillator knowledge according to level of seniority of personnel.

Doctors had the best knowledge with regard to the indications for both defibrillation (49\%) and synchronised cardioversion (18\%).

\section{Discussion}

Early defibrillation improves the outcome of patients presenting in cardiac arrest - for every minute's delay in defibrillation, survival decreases by $7-15 \% .{ }^{1,3}$ The defibrillator plays a central role in patients presenting with shockable arrest rhythms (VF and pulseless VT); however, to have the maximum impact on patient outcomes, defibrillation must be done quickly and correctly. ${ }^{1}$ Selecting the correct Joule setting 


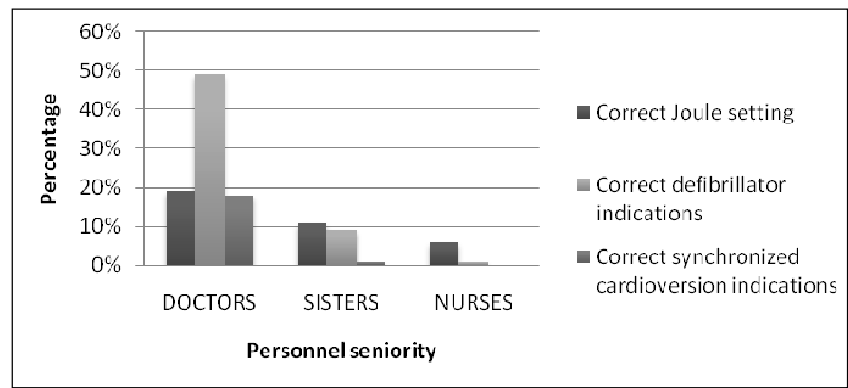

Fig. 1. Comparison of defibrillator knowledge according to seniority.

is an essential component of this process. Worryingly, in this study only $14 \%$ of personnel knew the correct Joule setting for their EC's defibrillator. This may lead to unnecessary delays or incorrect dosages and adversely affect survival.

All EC resuscitation equipment should be regularly checked to ensure its good working condition; this is especially applicable to defibrillators. ${ }^{4}$ The Emergency Medical Society of South Africa (EMSSA) recommends daily defibrillator testing as a national standard. ${ }^{5}$ The outcome of the testing should also be documented to ensure that it is working when it is required. In the absence of documentation of testing, the facility would be defenceless in a medicolegal case.

Anecdotally, in Western Cape ECs the main responsibility for the use of defibrillators lies with doctors. Comments on the questionnaires indicated that most nurses perceived that the use of defibrillators falls outside their scope of practice, although no formal documentation was found to validate this.

Defibrillators are most likely to be used in an EC or intensive care setting, or by cardiac arrest teams. In the vast majority of Western Cape hospitals, this means that EC personnel will most often make use of defibrillators, suggesting that their use of and knowledge of defibrillators should be better than their colleagues in a non-emergency environment. ${ }^{6}$ Only $18 \%$ knew the correct indications for defibrillation: even allowing for the argument that defibrillation is outside of a nurse's scope of practice, all doctors working in an EC should know these indications, but only $49 \%$ did so. This is despite $88 \%$ having received training, and indicates a serious problem with skill retention. Although training is important, ${ }^{7,8}$ retention of knowledge is more important: regular updates should occur to improve retention of knowledge and skills., ${ }^{9,10}$

There are two other standard emergency indications for defibrillator use: pacing and synchronised cardioversion. Both form part of the Western Cape Packages of Care ${ }^{11}$ at all levels, and the skills and knowledge should therefore be present.

\section{Limitations}

As this was a self-reported survey, the results may not exactly represent current practices. However, it is probable that it reflects the best-case scenario, as respondents tend to overstate their answers in anonymous questionnaires. If this is the case, the results may be even more concerning, adding to the urgency for intervention.

\section{Conclusion}

Defibrillators are life-saving and their early use improves survival of patients presenting with sudden cardiac arrest due to shockable rhythms if they are used safely and correctly. However, adequate knowledge and skills are of paramount importance to prevent unnecessary delays that may impact on patient outcome. Most EC personnel in the Western Cape do not have adequate levels of defibrillator knowledge.

Nurse-initiated defibrillation can be life-saving, and nursing personnel must be encouraged to add defibrillation to their scope of practice as an expected rather than an extended skill. This will need a change in mind-set on the part of many nurses in the provincial setting.

To ensure maximum patient benefit from defibrillator use, hospitals should aim to standardise equipment (this needs to be driven from Government level), ensure daily testing that is logged in a dedicated $\log$, and mandate regular updates and training in defibrillator skills for staff.

\section{Conflicts of interest: None.}

References

1. Bossaert L, Callanan V, Cummins RO. Early defibrillation: An advisory statement by the Advanced Life Support Working Group of the International Liaison Committee on the Advanced Life Support Working Group

2. Peberby MA, Kaye W, Ornato JP. Cardiopulmonary resuscitation of adults in the hospital: a report of 14720 cardiac arrests from the National Registry of Cardiopulmonary Resuscitation. Resuscitation 2003; 58: 297-308.

3. Chan PS, Krumholz HM, Nichol G, et al. Delayed time to defibrillation after in-hospital cardiac arrest. N Engl J Med 2008; 358: 9-17.

4. Cummins RO, Chesemore K, White RD. Defibrillator failures. Causes of problems and recommendations for improvement. Defibrillator Working Group. JAMA 1990; 264(8): 10191025

5. Emergency Medical Society of South Africa (EMSSA). Emergency Centre Defibrillation. Practice guideline EM008. January 2009. http:/ /www.emssa.org.za (accessed 15 January 2010).

6. Hou S, Chern C, How C, Wang L, Huang C, Lee C. Is ward experience in resuscitation effort related to the prognosis of unexpected cardiac arrest? J Chin Med Assoc 2007; 70(9): 385-390.

7. Joint statement from the Royal College of Anaesthetists, Royal College of Physicians of London, Intensive Care Society and Resuscitation Council (UK). Cardiopulmonary Resuscitation: Standards for clinical practice and training. October 2004. www.resus.org.uk (accessed 23 January 2010).

8. Stross JK. Maintaining competency in advanced cardiac life support skills. JAMA 1983; 249: 3339-3341.

9. Hamilton R. Nurses' knowledge and skill retention following cardiopulmonary resuscitation training: a review of literature. J Adv Nurs 2005; 51(3): 288-297.

10. Semeraro F, Signore L, Cerchiari EL. Retention of CPR performance in anaesthetists. Resuscitation 2006; 68(1): 101-108.

11. Western Cape Department of Health Hospital Package of Care. Cape Town: Provincial Government of the Western Cape, February 2009.

Accepted 23 March 2010. 


\section{Appendix. Defibrillation Questionnaire}

Job description

Is there a defibrillator in the EC?

Is there a defibrillator in the Hospital?

Is there an Automated External Defibrillator (AED)?

Are daily defibrillation tests done?

Who does above?

Have you had defibrillator Training?

Have you used a defibrillator before?

When was the last time you defibrillated?

Have you seen a defibrillator used before?

Do you have a mono- or biphasic defibrillator?

What Joule setting do you use?

What are the indications for defibrillation?

What are the indications for synchronized cardioversion?

Are there pacing leads and pads in the EC?

Are there pacing leads and pads in Hospital?

Have you paced before?

Have you seen pacing done before?

Other Comments

$\mathrm{EC}=$ Emergency Centre / Casualty / Trauma please mark correct box for each question

Comments



Sister
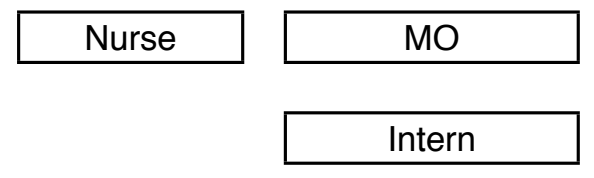

Reg
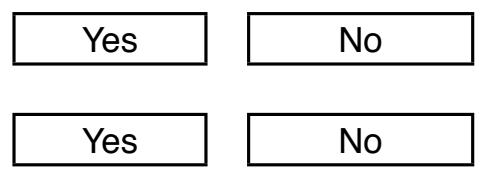

Yes
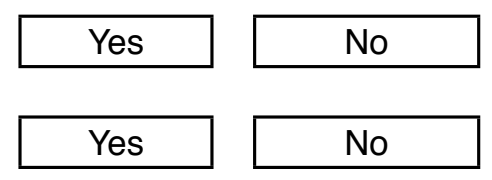

$\mathrm{Sr}$
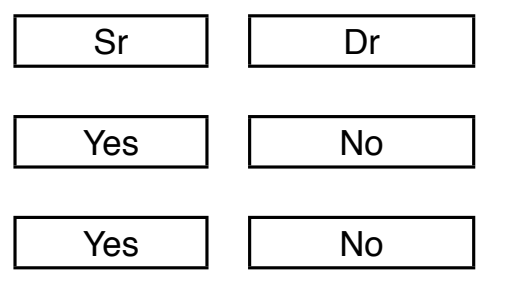

1 week

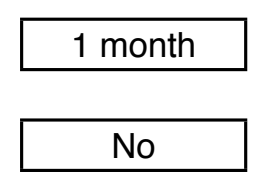

Yes

Mono

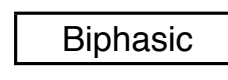

Don't know

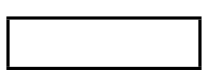

Don't know

\section{COSMO}
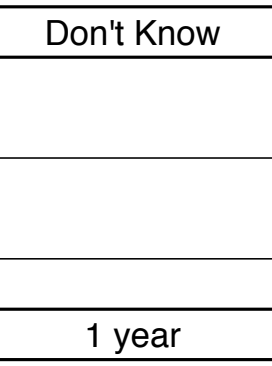

Not sure 